\title{
Herpes zoster in the ophthalmic branch of the trigeminal ganglia obscuring cavernous sinus thrombosis due to Streptococcus constellatus subsp. constellatus - A case report -
}

Received October 14, 2019

Revised December 5, 2019

Accepted December 11, 2019

\section{Ji Hye Lee, Hyun Joo Heo, Ki Man Kim, Han Gyeol Lee, Seung Min Baek, and Da Wa Jung}

Department of Anesthesiology and Pain Medicine, Presbyterian Medical Center, Jeonju, Korea

Background: Herpes zoster ophthalmicus (HZO) is an infectious disease that results from the reactivation of latent varicella zoster virus in the ophthalmic branch of the trigeminal ganglia. HZO manifests with herpes zoster-like symptoms such as rash with or without signs of ocular involvement. Cavernous sinus thrombosis (CST) is a life-threatening condition accompanied by signs and symptoms involving the eyes and the cranial nerves.

Case: We report a case of septic cavernous sinus thrombosis (caused by Streptococcus constellatus subsp. constellatus) which was masked by the simultaneous occurrence of $\mathrm{HZO}$ in this patient, resulting in delayed diagnosis.

Conclusions: CST may be obscured by HZO, prompt diagnosis and treatment is necessary when such case arrive.

Keywords: Cavernous sinus thrombosis; Herpes zoster; Streptococcus constellatus; Trigeminal ganglion.

\author{
Corresponding author \\ Ji Hye Lee, M.D. \\ Department of Anesthesiology and \\ Pain Medicine, Presbyterian Medical \\ Center, 365 Seowon- ro,Wansan-gu, \\ Jeonju 54987, Korea \\ Tel: 82-63-230-1593 \\ Fax: 82-63-230-8919 \\ E-mail: o2ljh@hanmail.net
}




\section{CASE REPORT}

A 44-year-old man was brought to a primary care hospital with a rash and vesicle on the left side of his forehead since 3 days. Moreover, he had a headache since 6 days. Serologic tests for VZV (immunoglobulin M [IgM] and immunoglobulin $\mathrm{G}$ [IgG]) were conducted using the enzyme-linked immunosorbent assay in the primary care hospital. The patient tested positive for VZV IgG and IgM. He was diagnosed with $\mathrm{HZO}$, and treated with antiviral drugs and analgesics. Before hospitalization, he had continuous fever $\left(38^{\circ} \mathrm{C}\right.$ and higher). After three days of hospitalization, the patient presented with worsening of left-sided frontal headache, nausea, blurred vision, and diplopia. He was transferred to our hospital for further evaluation and treatment.

The patient was alert but febrile $\left(38.3^{\circ} \mathrm{C}\right)$ when he arrived at our hospital. There was no marked facial or periorbital swelling, except for the crust on his forehead. The cardiopulmonary and neurological findings were normal. His heart rate, blood pressure, and respiratory rate were 78 beats/min, 110/60 mmHg, and 20 breaths/min. An ophthalmologist examined his eyes and the visual acuity was $6 / 12$ bilaterally. There were no abnormalities in the ocular tissues (cornea, conjunctiva, sclera, uvea, or retina) and no visual field defects. We suspected that his horizontal diplo- pia and lateral rectus muscle paresis in the left eye were because of 6th cranial nerve (abducens nerve) palsy. There was no evidence of infection in the facial region (except HZO), respiratory tract, urinary tract, or on the skin. Thus, the study focused on fever and cause of 6th cranial nerve palsy.

The white blood cell count was normal $\left(10,100 \mathrm{~mm}^{3}\right)$, but the erythrocyte sedimentation rate $(120 \mathrm{~mm} / \mathrm{h})$ and C-reactive protein level $(10.17 \mathrm{mg} / \mathrm{dl})$ were elevated. Blood culture was performed; however, the patient did not provide consent for a cerebrospinal fluid (CSF) analysis. The brain MRI revealed lateral bulging and irregular marginal enhancement in the cavernous sinus, which is consistent with bilateral CST and superior ophthalmic vein thrombosis (Fig. 1).

We admitted the patient to the intensive care unit and asked a neurologist and an infectious medicine doctor to assist with the treatment. Patient's history was taken again and he was kept under observation during the course of the treatment. Although he had no medical history of diabetes mellitus, hypertension, or etc., he did undergo dental treatment about 3 months ago and had experienced pain in the left maxillary area approximately 1 month ago. Given the dental history, high body temperature, and aggravated symptoms, we started his antibiotic treatment despite the antiviral treatment that he had received at the primary care
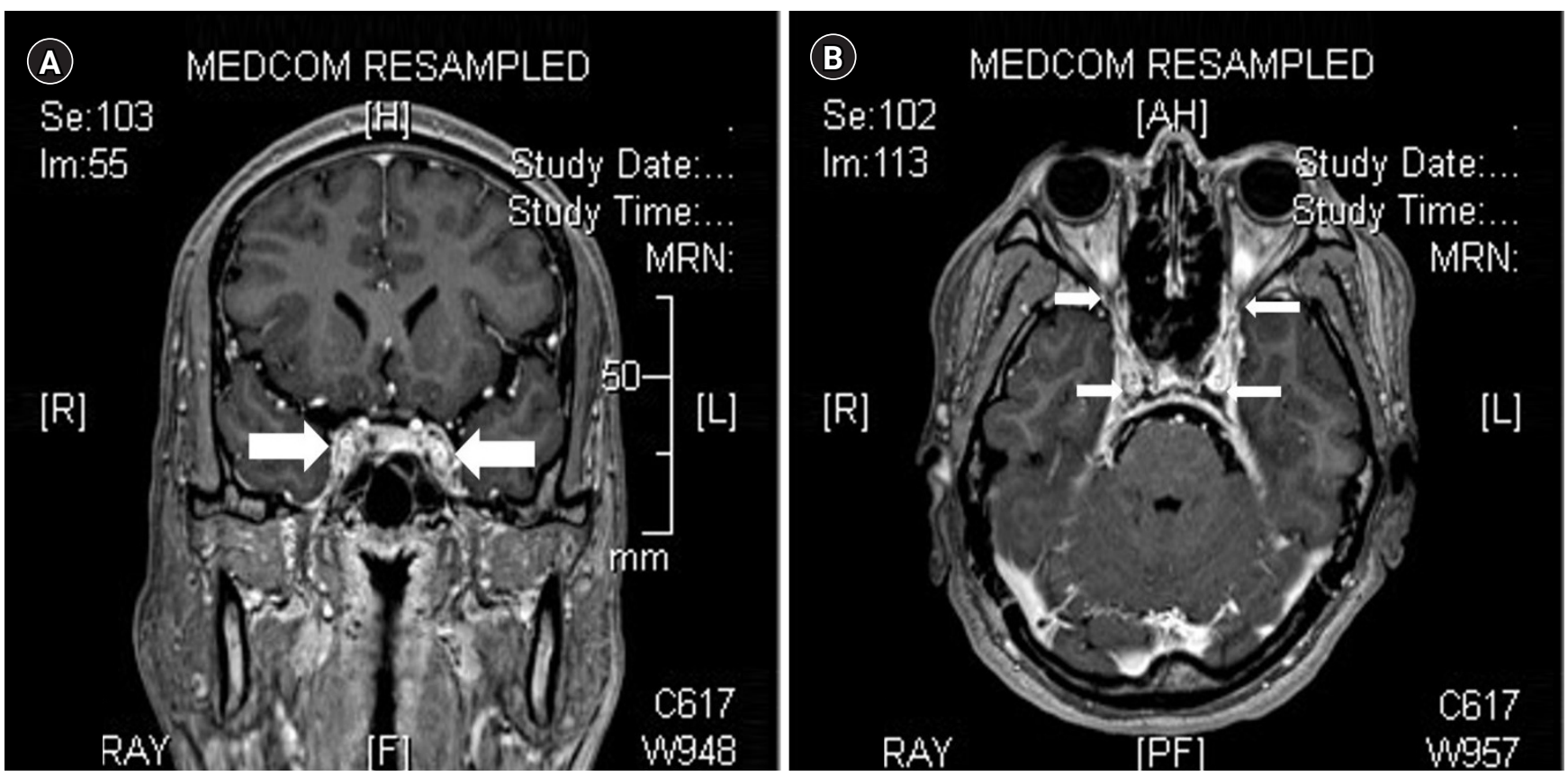

Fig. 1. Both superior ophthalmic veins presenting with engorgement and thrombus in the posterior region (A: axial, B: coronal view). 
hospital. Second-generation cephalosporin was administered until we received his blood culture results. Streptococcus constellatus subsp. constellatus was detected in the blood culture, therefore we switched from cephalosporin to clindamycin. We performed echocardiography to detect sings of infective endocarditis; however, it did not reveal any special findings. Antithrombotic therapy was performed with low molecular weight heparin. After 7 days of treatment in the intensive care unit, the symptoms improved, and the patient was discharged after being transferred to the general room. No complications were observed during one year of follow-up since the patient was discharged.

\section{DISCUSSION}

Herpes zoster is frequently encountered in primary care hospitals. The lifetime risk of herpes zoster is estimated to be about $30 \%$ and, HZO appears in $20 \%$ of these cases, with risks in about $1-4 \%$ throughout life $[1,2]$. Herpes zoster patients complain of systemic symptoms of influenza-like illness, such as fatigue, malaise, and low-grade fever, prior to the development of a unilateral rash. A rash and a vesicle characteristically appear along the nerve segment where the virus reactivates, but like zoster sine herpete, the occasional skin lesions do not appear. HZO presents with the general symptoms of herpes zoster infection and the eruption of vesicle and cluster of papules in the trigeminal nerve dermatome divisions located on the forehead, upper eyelids, eyes, and nose. Ocular manifestations may occur, including ptosis and swelling, injected appearance, petechial hemorrhage in the conjunctiva, keratitis and scarring ulcer in the cornea, uveitis, and acute retinal necrosis. In addition, patients with HZO may manifest symptoms of optic neuritis, swelling, edematous optic nerve, and oculomotor palsy [1,2]. The ocular symptoms in HZO can be explained by the inflammation of the ocular nerve through cavernous sinus caused by the reactivation of VZV in the trigeminal ganglion.

The signs and symptoms of VZV primary infection, also known as chicken pox, are attributed to vasculopathy, which may manifest in the form of transient ischemic attacks, ischemic or hemorrhagic stroke, aneurysm, sinus thrombosis, giant cell arteritis, and granulomatous aortitis. CST is a type of cerebral venous sinus thrombosis (CVST). The CVST is mostly reported in pediatric patients with VZV primary infection accompanied with purpura fulminans.
By contrast, the VZV primary infection is rare in adults and has more severe symptoms [7-9]. Herpes zoster, that is reactivation of VZV, has no reported cases of CVST. Herpes zoster is, however, a risk factor for stroke and myocardial infarction. Moreover, the incidence of stroke increases in patients who have a history of HZO [10].

Cavernous sinus is connected to the dural venous sinus in the midline of the skull with direct or indirect nasal, ethmoidal, and sphenoidal sinus veins. CST may be septic or aseptic. Causes of aseptic CST are trauma, surgery, hematological disorder, malignancy, or it could be idiopathic in origin [5]. Septic CST occurs because of the infection spreading through the ethmoidal or sphenoidal sinuses, or from teeth, ear, or orbit through the venous system. Septic CST is more common than aseptic CST [3,5]. In the current study, the patient's blood culture detected Streptococcus constellatus subsp. constellatus, which belongs to the milleri group. Streptococci in this group are part of the resident flora of the oral cavity and the respiratory tract. However, they may cause suppurative and deep-seated infections via the venous system. The incidence of CVST is less than $1 \%$ of all strokes [4]. The exact incidence rate of CST is not known, but it is rare like CVST. Infection, pregnancy, receiving oral contraceptives, thrombophilic disorders, and obesity could contribute to increased CST risk. CST is a life-threatening condition [3-6]. Although the symptoms of CST may vary, in most cases, the symptoms appear in relation to the anatomical structures near the cavernous sinus, such as cranial nerves, orbit, and eyes, because their venous drainage is in the cavernous sinus. Typical symptoms of CST are headache, unilateral periorbital edema, photophobia, and bulging eyeballs. In addition, involvement of the cranial nerve may result in palsy or sensory nerve injury, such as sensory deficit of the ophthalmic and maxillary branches of the 5th cranial nerve [3-6].

The signs and symptoms of HZO and CST are similar. In addition, HZO can cause vasculopathy, such as transient ischemic attacks and ischemic or hemorrhagic stroke [7]. In our patient, skin lesions occurred at the trigeminal nerve V1 site, three days before the visit, as seen in cases of HZO, along with fever and myalgia. The patient was diagnosed with HZO in the primary care hospital and was treated accordingly. However, even after the treatment, he had persistent headache and high fever and was transferred to our hospital to rule out meningitis and stroke. The patient did not provide consent for a CSF study, but blood culture was performed because of high fever, and CST was confirmed on 
MRI. The preceding dental treatment may have been the cause of CST in this patient.

Herpes zoster-associated vasculopathy is treated with antiviral agents. However, infectious CST is treated with high-dose antibiotics. CST has a high mortality rate; therefore, prompt treatment is necessary. We started the treatment for CST immediately while continuing, the HZO treatment. HZO misdiagnosis may delay the diagnosis of CST because vasculopathy makes it difficult to differentiate the symptoms of CST and HZO. If high fever, severe headache, and eye symptoms are observed with HZO, the patient history should be accurately evaluated to exclude CST by performing not only CSF analysis but also MRI, blood culture, etc.

In conclusion, CST is a life-threatening condition and its diagnosis may be obscured by HZO. Therefore, prompt diagnosis and treatment are necessary in such cases.

\section{CONFLICTS OF INTEREST}

No potential conflict of interest relevant to this article was reported.

\section{AUTHOR CONTRIBUTIONS}

Conceptualization: Ji Hye Lee, Hyun Joo Heo. Data acquisition: Ki Man Kim, Han Gyeol Lee, Seung Min Baek, Da Wa Jung. Supervision: Ji Hye Lee. Writing-original draft: Ji Hye Lee. Writing_review \& editing: Ji Hye Lee, Hyun Joo Heo.

\section{ORCID}

Ji Hye Lee, https://orcid.org/0000-0003-3269-3844 Hyun Joo Heo, https://orcid.org/0000-0003-2507-6629

Ki Man Kim, https://orcid.org/0000-0002-4257-9167
Han Gyeol Lee, https://orcid.org/0000-0001-7835-0172 Seung Min Baek, https://orcid.org/0000-0003-0290-0208

Da Wa Jung, https://orcid.org/0000-0002-1566-7903

\section{REFERENCES}

1. Ragozzino MW, Melton LJ 3rd, Kurland LT, Chu CP, Perry HO. Population-based study of herpes zoster and its sequelae. Medicine (Baltimore) 1982; 61: 310-6.

2. Johnson JL, Amzat R, Martin N. Herpes zoster ophthalmicus. Prim Care 2015; 42: 285-303.

3. Coutinho JM. Cerebral venous thrombosis. J Thromb Haemost 2015; 13 Suppl 1: S238-44.

4. Einhäupl K, Stam J, Bousser MG, De Bruijn SF, Ferro JM, Martinelli I, et al.; European Federation of Neurological Societies. EFNS guideline on the treatment of cerebral venous and sinus thrombosis in adult patients. Eur J Neurol 2010; 17: 1229-35.

5. Lai PF, Cusimano MD. The spectrum of cavernous sinus and orbital venous thrombosis: a case and a review. Skull Base Surg 1996; 6: 53-9.

6. Weerasinghe D, Lueck CJ. Septic cavernous sinus thrombosis: case report and review of the literature. Neuroophthalmology 2016; 40: 263-76.

7. Siddiqi SA, Nishat S, Kanwar D, Ali F, Azeemuddin M, Wasay M. Cerebral venous sinus thrombosis: association with primary varicella zoster virus infection. J Stroke Cerebrovasc Dis 2012; 21: 917.e1-4.

8. Paul G, Paul BS, Singh G. Unseen face of varicella-zoster infection in adults. Indian J Crit Care Med 2016; 20: 731-4.

9. Khan R, Yasmeen A, Pandey AK, Al Saffar K, Narayanan SR. Cerebral venous thrombosis and acute pulmonary embolism following varicella infection. Eur J Case Rep Intern Med 2019; 6: 001171.

10. Nagel MA, Jones D, Wyborny A. Varicella zoster virus vasculopathy: the expanding clinical spectrum and pathogenesis. J Neuroimmunol 2017; 308: 112-7. 